\title{
A Simple Quantum Mechanics Way to Simulate Nanoparticles and Nanosystems without Calculation of Wave Functions
}

\author{
Victor G. Zavodinsky ${ }^{1}$ and Olga A. Gorkusha² \\ ${ }^{1}$ Institute of Materials of the Far Eastern Branch of the Russian Academy of Sciences, Tikhookeanskaya str. 153, \\ Khabarovsk 680042, Russia \\ ${ }^{2}$ Institute of Applied Mathematics of the Far Eastern Branch of the Russian Academy of Sciences, Dzerzhinskogo str. 54, \\ Khabarovsk 680000, Russia \\ Correspondence should be addressed to Victor G. Zavodinsky, vzavod@mail.ru
}

Received 19 June 2012; Accepted 9 July 2012

Academic Editors: J. R. Vargas Garcia and S. Wang

Copyright ( 2012 V. G. Zavodinsky and O. A. Gorkusha. This is an open access article distributed under the Creative Commons Attribution License, which permits unrestricted use, distribution, and reproduction in any medium, provided the original work is properly cited.

It is shown that the variation principle can be used as a practical way to find the electron density and the total energy in the frame of the density functional theory (DFT) without solving of the Kohn-Sham equation. On examples of diatomic systems $\mathrm{Si}_{2}, \mathrm{Al}_{2}$, and $\mathrm{N}_{2}$, the equilibrium interatomic distances and binding energies have been calculated in good comparison with published data. The method can be improved to simulate nanoparticles containing thousands and millions atoms.

\section{Introduction}

It is well known [1] that the electron ground state energy $E^{\mathrm{el}}$ of a quantum system may be found by minimization of the energy functional which depends on the total electron density $\rho$ as follows:

$$
\begin{aligned}
E^{\mathrm{el}}[\rho]= & E^{t}[\rho]+E^{\mathrm{ex}}[\rho]+E^{\mathrm{cor}}[\rho]+E^{\mathrm{H}}[\rho] \\
& -\int V(\vec{r}) \rho(\vec{r}) d^{3} \vec{r},
\end{aligned}
$$

where $V, E^{t}, E^{\mathrm{ex}}, \mathrm{E}^{\mathrm{cor}}$, and $E^{\mathrm{H}}$ are an external potential, the kinetic, exchange, correlation, and Hartree energies, correspondingly, and $E^{\mathrm{H}}$ is given as follows:

$$
E^{\mathrm{H}}[\rho]=\frac{1}{2} \int \frac{\rho(\vec{r}) \rho\left(\overrightarrow{r^{\prime}}\right)}{\left|\vec{r}-\vec{r}^{\prime}\right|} d^{3} \vec{r} d^{3} \vec{r} .
$$

The first attempt to study the quantum system without wave functions was made by Thomas and Fermi [2, 3]. They considered the interrelation of the electron density, the electronic kinetic energy, and the electrostatic potential of an isolated atom and have found a simple one-dimensional equation for the potential. Then their idea has been developed by a number of authors [4-6], however still nowadays this theory is used only for single-atomic and other radial symmetric systems, or for jellium-approached ones [7-9]. Our work demonstrates a possibility to calculate the total energy and the electron density for neutral many-atomic systems directly from the minimization of the energy functional equation (1).

\section{Results and Discussion}

The variation principle together with the constant number of electrons $N$ gives

$$
\frac{\delta E[\rho]}{\delta \rho}=\mu \quad \text { with } \int \rho(\vec{r}) d^{3} \vec{r}=N
$$
$\rho(\vec{r})$

Thus we have the equation for finding the equilibrium

$$
\begin{aligned}
F[\rho] \equiv \frac{\delta E[\rho]}{\delta \rho}= & -V(\vec{r})+\varphi(\vec{r})+\mu_{t}(\rho)+\mu_{\mathrm{ex}}(\rho) \\
& +\mu_{\text {cor }}(\rho)-\mu=0,
\end{aligned}
$$

where $\varphi(\vec{r})=\int\left(\rho\left(\overrightarrow{r^{\prime}}\right) /\left|\vec{r}-\overrightarrow{r^{\prime}}\right|\right) d^{3} \overrightarrow{r^{\prime}}, \mu_{t}(\rho)=\delta T[\rho] / \delta \rho$, $\mu_{\mathrm{ex}}(\rho)=\delta E^{\mathrm{ex}}[\rho] / \delta \rho$, and $\mu_{\mathrm{cor}}(\rho)=\delta E^{\mathrm{cor}}[\rho] / \delta \rho$. 
Equation (4) may be solved using any iteration method if you can calculate all its components. For simplicity, we have limited our current consideration by the local density approximation (LDA), in which

$$
\begin{aligned}
\mu_{t} & =\frac{1}{5}\left(3 \pi^{2} \rho\right)^{2 / 3}, \quad \varepsilon_{t}=\frac{3}{10}\left(3 \pi^{2}\right)^{2 / 3} \rho^{5 / 3}, \\
\mu_{\mathrm{ex}} & =-\left(\frac{3 \rho}{\pi}\right)^{1 / 3}, \quad \varepsilon_{\mathrm{ex}}=-\frac{3}{4 \pi}\left(3 \pi^{2}\right)^{1 / 3} \rho^{4 / 3} .
\end{aligned}
$$

The correlation potential $\mu_{\text {cor }}$ and the correlation energy $\mathcal{E}_{\text {cor }}$ were taken as it was proposed in works $[10,11]$. Namely,

$$
\begin{gathered}
\varepsilon_{\mathrm{cor}}=\frac{\gamma}{\left(1+\beta_{1} \sqrt{r_{s}}+\beta_{2} r_{s}\right)}, \\
\mu_{\mathrm{cor}}=\left(1-\frac{r_{s}}{3} \frac{d}{d r_{s}}\right) \varepsilon_{\mathrm{cor}}=\varepsilon_{\mathrm{cor}} \frac{1+(7 / 6) \beta_{1} \sqrt{r_{s}}+(4 / 3) \beta_{2} r_{s}}{1+\beta_{1} \sqrt{r_{s}}+\beta_{2} r_{s}},
\end{gathered}
$$

for $r_{s}=(4 / 3 \pi \rho)^{-1 / 3} \geq 1$.

And

$$
\begin{gathered}
\varepsilon_{\mathrm{cor}}=A \ln r_{s}+B+C r_{s} \ln r_{s}+D r_{s}, \\
\mu_{\mathrm{cor}}=A \ln r_{s}+\left(B-\frac{1}{3} A\right)+\frac{2}{3} C r_{s} \ln r_{s}+\frac{1}{3}(2 D-C) r_{s},
\end{gathered}
$$

for $r_{s}=(4 / 3 \pi \rho)^{-1 / 3}<1$.

Here $A=0.0311, B=-0.048, C=0.002, D=-0.0116$, $\gamma=-0.1423, \beta_{1}=1.0529$, and $\beta_{2}=0.3334$ (in a.e.).

The well-known Newton's iteration method was used to find $\rho(\vec{r})$ as a self-consisted solution for (4):

$$
\rho_{i+1}=\rho_{i}-\frac{F\left[\rho_{i}\right]}{d F\left[\rho_{i}\right] / d \rho_{i}} .
$$

The main problem is to calculate the Coulomb potential $\varphi(\vec{r})$ for a many-atomic system, and it is seems that this is a main reason why there was a little progress on this way. However, at the last years, the efficient methods for calculation of Coulomb integrals were developed. Namely, we used the supercell-cut-off technique $[12,13]$ and the fast Fourier transformation code [14].

The second problem is to operate with all-electrons atomic potentials which have strong singularities leading to very sharp density peaks and, as a result, to impossibility to calculate the Coulomb potential correctly through the Fourier expansion with limited number of plane waves. Therefore, we worked within the frame of a pseudopotential version of the DFT theory, constructing pseudopotentials using FHI98PP code [15] and taking into account only valence electrons. For simplicity we have considered only diatomic systems $\left(\mathrm{Si}_{2}, \mathrm{Al}_{2}, \mathrm{~N}_{2}\right)$, thus the total external potential $V(\vec{r})$ was calculated as a sum of pseudopotentials $V_{p}\left(\vec{r}-\vec{R}_{1}\right)$ and $V_{p}\left(\vec{r}-\vec{R}_{2}\right)$ centered on the positions of two atoms, $\vec{R}_{1}$ and $\vec{R}_{2}$. The sum of pseudoatomic densities, $\rho_{p}(\vec{r}-$ $\left.\vec{R}_{1}\right)+\rho_{p}\left(\vec{r}-\vec{R}_{2}\right)$, was used as a start density for the Newton's iteration procedure. To find the equilibrium interatomic
TABLE 1: The equilibrium distances and binding energies for $\mathrm{Si}_{2}$, $\mathrm{Al}_{2}$, and $\mathrm{N}_{2}$. The published data is shown in brackets.

\begin{tabular}{lccc}
\hline & $\mathrm{Si}_{2}$ & $\mathrm{Al}_{2}$ & $\mathrm{~N}_{2}$ \\
\hline$d_{0}, \AA$ & $2.2\left(2.27^{\mathrm{a}}\right)$ & $2.6\left(2.51^{\mathrm{b}}\right)$ & $1.1\left(1.098^{\mathrm{c}}\right)$ \\
$E_{\mathrm{b}}, \mathrm{eV}$ & $3.3\left(3.07^{\mathrm{a}}\right)$ & $1.4\left(1.55^{\mathrm{b}}\right)$ & $7.3\left(9.42^{\mathrm{d}}\right)$ \\
\hline
\end{tabular}

${ }^{\mathrm{a}}$ Calculated [16]; ${ }^{\mathrm{b}}$ calculated [17]; ${ }^{\mathrm{c}}$ experimental [18]; ${ }^{\mathrm{d}}$ experimental [19].

distance $\left(d_{0}\right)$ we considered the total energy $E^{\text {tot }}$ as a sum of the electron energy, $E^{\mathrm{el}}$, and the energy of the "ion-ion" repulsion, $E^{\text {rep }}$, that is, the interaction energy of positivecharged point nucleus

$$
E^{\text {rep }}=\frac{Z_{v}^{2}}{\left|\vec{R}_{1}-\vec{R}_{2}\right|}
$$

where $Z_{v}$ is the number of valence electrons: $Z_{v}(\mathrm{Si})=4$, $Z_{v}(\mathrm{Al})=3$, and $Z_{v}(\mathrm{~N})=5$.

With the self-consisted $\rho(\vec{r})$, obtained from (4), we calculated the total energy $E^{\text {tot }}$ as a function of the interatomic distance $d=\left|\vec{R}_{1}-\vec{R}_{2}\right|, E^{\text {tot }}=E^{\mathrm{el}}+E^{\mathrm{rep}}$.

Values of the equilibrium distances $d_{0}$ and binding energies $E_{\mathrm{b}}$ for studied species are listed in Table 1 comparing with published data.

\section{Summary}

Our results show that the calculated equilibrium binding energies and distances are close to published data; thus we can be sure that this approach may be used for modeling of huge particles, probably up to million atoms.

Our consideration is limited by the spin-restricted case; however, we believe that the spin polarization can be included as well as the general gradient approximation.

The main advantage of the developed method consists in the independence of the calculation time from the number of electrons; it depends linearly on the volume of the system, or in other words on the number of atoms. Certainly, it does not give us electron states, but they can be easily calculated through the usual Kohn-Sham technique if we know the electron density. We also believe that the proposed approach can be successfully improved in future for all-electrons atoms.

\section{References}

[1] P. Hohenberg and W. Kohn, "Inhomogeneous electron gas," Physical Review, vol. 136, no. 3, pp. B864-B871, 1964.

[2] L. H. Thomas, "The calculation of atomic field," Proceedings of the Cambridge Philosophical Society, vol. 23, pp. 542-548, 1927.

[3] E. Fermi, "Un metodo statistico per la determinazione di alcune prioprietà dell'atomo," Rendiconti: Accademia Nazionale dei Lincei, vol. 6, pp. 602-607, 1927.

[4] P. A. M. Dirac, "Note on exchange phenomena in the Thomas atom," Proceedings of the Cambridge Philosophical Society, vol. 26, pp. 376-385, 1930. 
[5] L. Hedin and B. I. Lundqvist, "Explicit local exchange-correlation potentials," Journal of Physics C, vol. 4, no. 14, pp. 2064-2083, 1971.

[6] D. A. Kirzhnits, "Quantum corrections to the ThomasFermi equation," Soviet Physics, Journal of Experimental and Theoretical Physics Letters, vol. 5, pp. 64-72, 1957, Pis'ma v Zhurnal Eksperimental'noi i Teoreticheskoi Fiziki, vol. 32, pp. 115-123, 1957.

[7] B. M. Deb and P. K. Chattaraj, "Thomas-Fermi-type method for the direct calculation of electronic densities and properties of atoms and ions," Physical Review A, vol. 45, no. 3, pp. 14121419, 1992.

[8] V. Ya. Karpov and G. V. Shpatakovskaya, "Quasi classic calculation of the electron density distribution in some electronionic systems," Annual Moscow Workshop (Physics of Nonideal Plasmas), Moscow, 3-4 December 2002, http://zhurnal .ape.relarn.ru/articles/2002/191.pdf.

[9] E. Gaite, M. Leal, and E. Santos, "Study of interplanar binding in graphite by extended Thomas-Fermi theory," Physical Review B, vol. 31, no. 12, pp. 8226-8231, 1985.

[10] J. P. Perdew and A. Zunger, "Self-interaction correction to density-functional approximations for many-electron systems," Physical Review B, vol. 23, no. 10, pp. 5048-5079, 1981.

[11] D. M. Ceperley and B. J. Alder, "Ground state of the electron gas by a stochastic method," Physical Review Letters, vol. 45, no. 7, pp. 566-569, 1980.

[12] M. R. Jarvis, I. D. White, R. W. Godby, and M. C. Payne, "Supercell technique for total-energy calculations of finite charged and polar systems," Physical Review B, vol. 56, no. 23, pp. 14972-14978, 1997.

[13] C. A. Rozzi, D. Varsano, A. Marini, E. K. U. Gross, and A. Rubio, "Exact Coulomb cutoff technique for supercell calculations," Physical Review B, vol. 73, no. 20, Article ID 205119, 2006.

[14] M. Frigo and S. G. Johnson, "FFTW: an adaptive software architecture for the FFT," in Proceedings of the IEEE International Conference on Acoustics, Speech and Signal Processing (ICASSP'98), vol. 3, pp. 1381-1384, May 1998.

[15] M. Fuchs and M. Scheffler, "Ab initio pseudopotentials for electronic structure calculations of poly-atomic systems using density-functional theory," Computer Physics Communications, vol. 119, no. 1, pp. 67-98, 1999.

[16] D. Tomańek and M. A. Schluter, "Structure and bonding of small semiconductor clusters," Physical Review B, vol. 36, no. 2, pp. 1208-1217, 1987.

[17] V. Kumar and V. Sundararajan, "Ab initio molecular-dynamics studies of doped magic clusters and their interaction with atoms," Physical Review B, vol. 57, no. 8, pp. 4939-4942, 1998.

[18] K. P. Huber and G. Herzberg, Molecular Spectra and Molecular Structure IV Constants of Diatomic Molecules, VanNostrandReinhold, New York, NY, USA, 1979.

[19] S. J. Blanksby and G. B. Ellison, "Bond dissociation energies of organic molecules," Accounts of Chemical Research, vol. 36, no. 4, pp. 255-263, 2003. 

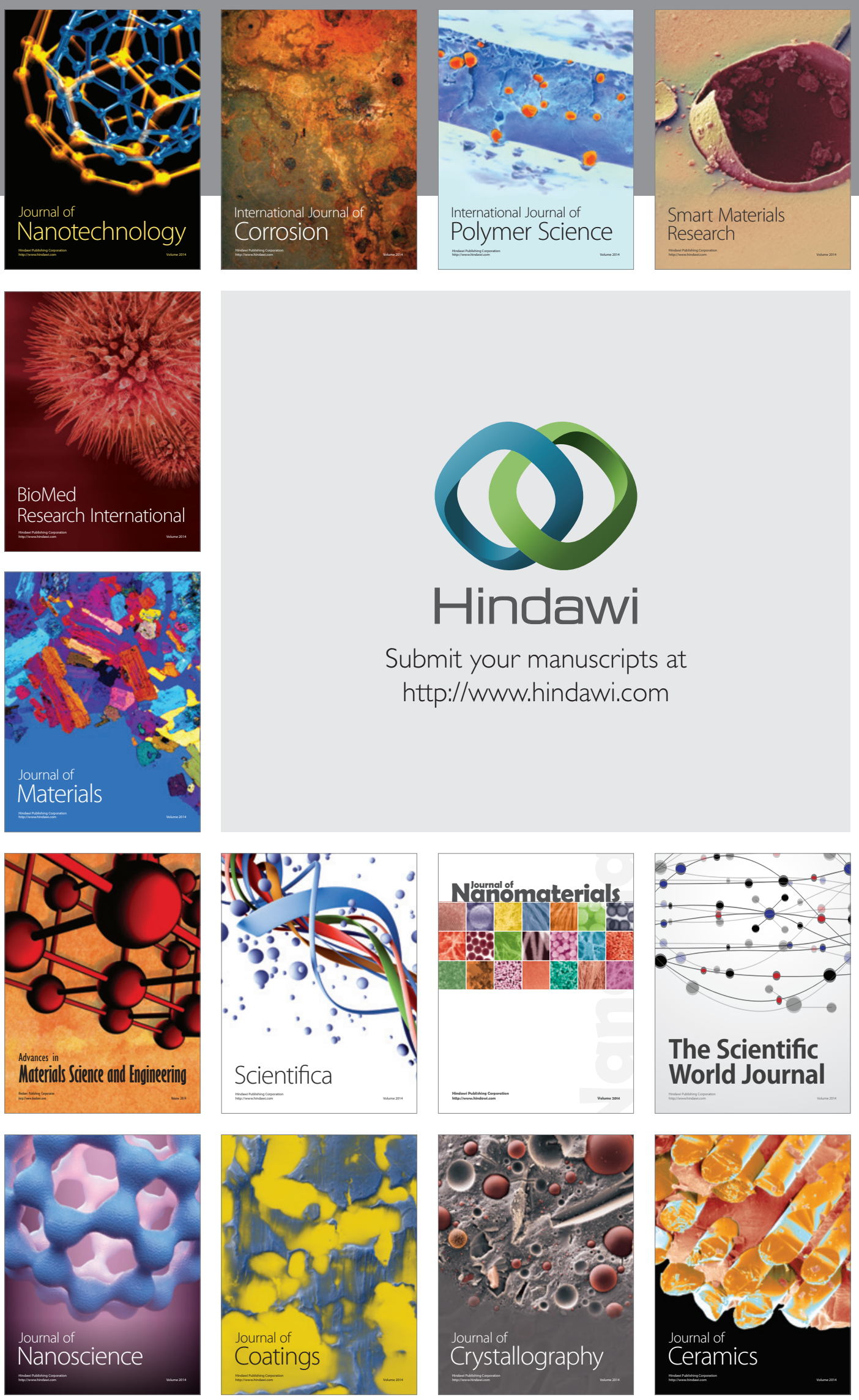

The Scientific World Journal

Submit your manuscripts at

http://www.hindawi.com

\section{World Journal}

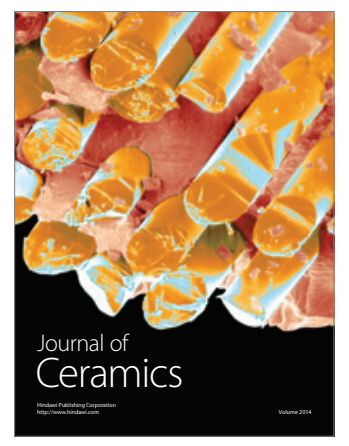

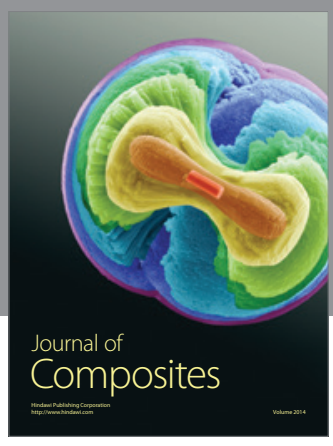
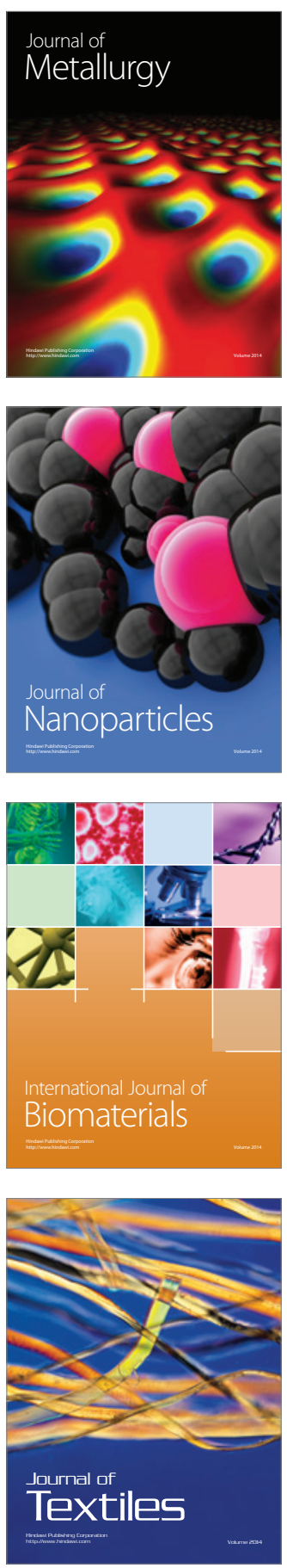